\title{
VIABILIDADE DE FOLÍCULOS PRÉ-ANTRAIS BOVINOS ISOLADOS DE OVÁRIOS APÓS VITRIFICAÇÃO
}

\author{
HÉlder Silva Luna ${ }^{1}$, Viviane Bento Silva ${ }^{2}$, Fernanda ARAujo Abreu² \\ ${ }^{1}$ Professor Doutor, Departamento de Biociências, Universidade Federal de Mato Grosso do Sul, Campo Grande, MS - \\ hluna@ceua.ufms.br \\ ${ }^{2}$ Acadêmicas do curso de Ciências Biológicas, Bolsista do Programa de Iniciação Científica, Universidade Federal de \\ Mato Grosso do Sul, Campo Grande, MS
}

O objetivo de presente estudo foi avaliar a viabilidade de foliculos pré-antrais bovinos isolados após processo de vitrificação. Ovários foram obtidos em abatedouro e submetidos a isolamento folicular. A suspensão obtida foi dividida em três partes, uma parte foi para o grupo controle, uma para o teste de toxicidade e a outra para procedimento de vitrificação. Para vitrificação, os folículos foram expostos por 1 minuto à solução de vitrificação 1, composta de $10 \%$ de etileno-glicol, $10 \%$ de DMSO em solução salina ( $\mathrm{NaCl}$ a $0,9 \%)$, e então 1 minuto à solução de vitrificação 2 , composta de $20 \%$ de etilenoglicol, 20\% de DMSO na mesma solução agora suplementada com 0,5 M de sacarose. Os folículos foram vitrificados com o uso de capilares de vidro e permaneceram vitrificados por 24 horas. Após desvitrificação, foram transferidos para solução a 0,25 M de sacarose por 2 minutos e então transferidos para solução de $0,15 \mathrm{M}$ de solução de sacarose por outros 2 minutos. Para o teste de toxicidade, os folículos foram somente expostos aos crioprotetores e analisados. Os foliculos pré-antrais foram classificados em não viáveis ou viáveis quando foram corados ou não com o azul de tripan, respectivamente. Diferenças $(\mathrm{P}<0,05)$ na viabilidade folicular foram observadas entre o controle $(93,6 \%)$ e folículos expostos $(64,1 \%)$ ou vitrificados (66\%). A porcentagem de folículos normais encontrados após exposição aos crioprotetores não apresentou diferença em relação aos vitrificados, indicando que a redução de viabilidade é atribuída aos efeitos tóxicos e não ao processo de vitrificação.

PALAVRAS-CHAVE: bovinos; crioprotetores; ovócitos; vitrificação.

\section{FOLLICULAR VIABILITY OF THE ISOLATED PREANTRAL FOLLICLES OF BOVINE OVARIES AFTER VITRIFICATION}

\begin{abstract}
The aim of this study was to evaluate isolated bovine preantral follicles after vitrification procedure. Ovaries were obtained at a local slaughterhouse and submitted to follicular isolation. From the obtained suspension, one part was destined to control group. The remaining suspension was divided into two parts, one to the toxicity test and the other one to the vitrification procedure. For vitrification, the follicles were exposed for $1 \mathrm{~min}$ to vitrification solution-1, composed of $10 \%$ ethylene glycol, $10 \%$ DMSO in saline solution $(\mathrm{NaCl} 0.9 \%)$, and then 1 min to vitrification solution-2, composed of $20 \%$ ethylene glycol, 20\% DMSO in the same saline solution

supplemented with $0.5 \mathrm{M}$ sucrose. The follicles were vitrified by use of glass capillaries and remained vitrified for $24 \mathrm{~h}$. After warming, they were transferred to a $0.25 \mathrm{M}$ sucrose solution for $5 \mathrm{~min}$ and then transferred to a 0.15 $\mathrm{M}$ sucrose solution for another $5 \mathrm{~min}$. For the toxicity test, the follicles were only exposed to cryoprotectants and analised. The preantral follicles were classified as nonviable orviable when they were stained or not stained by trypan blue, respectively. Differences $(\mathrm{P}<0.05)$ in follicular viability were observed among the control, nonvitrified group $(93.6 \%)$, and exposed $(64.1 \%)$ or vitrified follicles $(66 \%)$. The percentages of normal follicles found 
mínimo de 24 horas.

Para desvitrificação, os folículos foram transferidos, imediatamente após sua retirada do nitrogênio, para uma solução de sacarose a $0,25 \mathrm{M}$, onde permaneceram por 2 minutos e, em seguida, transferidos para uma solução de sacarose a $0,15 \mathrm{M}$ por mais 2 minutos e depois depositados em solução salina.

Para o teste de toxicidade, os folículos foram expostos aos mesmos crioprotetores e períodos de tempo do grupo criopreservado, porém sem vitrificação. Após a exposição, o liquido contendo os folículos foi centrifugado por 2 minutos a $1000 \mathrm{rpm}$, para a remoção dos crioprotetores - esse procedimento foi repetido duas vezes.

Para o teste de viabilidade, foi utilizado o corante azul de tripan na concentração de $0,4 \%$. As análises foram realizadas em microscópio óptico com aumento de $100 \mathrm{x}$, sendo considerados viáveis os folículos que permaneceram não corados e inviáveis os corados (GUPTA et al., 2002).
Para comparações entre os grupos, foi realizada análise de variância (ANOVA) e teste Tukey. Os valores foram significativos quando $\mathrm{P}<0,05$. Foram realizadas cinco repetições para cada tratamento (um ovário por repetição).

\section{RESULTADOS E DISCUSSÃO}

Uma grande quantidade de folículos préantrais podem ser recuperados de um único ovário bovino por meio de métodos mecânicos e enzimáticos (FIGUEIREDO et al., 1993). Na presente pesquisa, 584 folículos foram isolados dos ovários por método mecânico e distribuídos entre os três grupos estudados. A Tabela 1 apresenta a viabilidade dos folículos pré-antrais imediatamente após o isolamento, grupo controle (Grupo 1), exposição à solução crioprotetora (Grupo 2) e vitrificação (Grupo 3). Observou-se diferença $(93,6 \%)$ do Grupo 1 com os Grupos $2(64,1 \%)$ e 3 $(66 \%)$, mas não entre os dois últimos.

Tabela 1 - Viabilidade dos folículos pré-antrais no Grupo 1 (controle), Grupo 2 (exposto à solução crioprotetora) e no Grupo 3 (vitrificado)

\begin{tabular}{ccc}
\hline Grupos & Número de folículos analisados & Número de folículos viáveis $(\%)$ \\
\hline Grupo 1 & 220 & $206(93,6)^{\mathrm{a}^{*}}$ \\
Grupo 2 & 217 & $139(64,1)^{\mathrm{b}}$ \\
Grupo 3 & 147 & $97(66,0)^{\mathrm{b}}$ \\
\hline
\end{tabular}

${ }^{\text {*a-b }}$ Sobrescritos diferentes na mesma coluna indicam diferença significativa $(\mathrm{P}<0,05)$.

A vitrificação é um método que expõe as estruturas a serem criopreservadas a altas concentrações de crioprotetores e taxas de resfriamento quando comparado ao procedimento de congelação clássico. Por outro lado, evita a formação de cristais de gelo intracelular que são deletérios aos sistemas biológicos (YAVIN \& ARAV, 2007). GANDOLFI et al. (2006) compararam a vitrificação com o método convencional na criopreservação de fragmentos de tecido ovariano de humanos, bovinos e suínos. Observaram que o método de congelação clássico foi mais eficiente na preservação da integridade folicular, após análise histológica do tecido, do que a técnica de vitrificação. No presente trabalho, foram avaliados os efeitos da associação dos crioprotetores etileno-glicol, dimetilsulfóxido e sacarose e do processo de vitrificação na viabilidade de folículos pré-antrais bovinos isolados mecanicamente de tecido ovariano bovino, diferentemente da forma utilizada por GANDOLFI et al. (2006), em que os folículos foram vitrificados inclusos em fragmentos ovarianos.

Em relação aos testes de viabilidade, o azul de tripan tem sido utilizado por diversos pesquisadores na análise da integridade folicular, em particular da membrana de folículos criopreservados (DEMIRCI et al., 2001; AMORIM et al., 2003). Observou-se, no estudo atual, uma redução na viabilidade dos folículos expostos à solução crioprotetora (Grupo 2; 64\%) e vitrificados (Grupo 3 ; 66\%) quando comparados ao Grupo controle (grupo 1; 93,6\%); entretanto, a redução da viabilidade é atribuída aos efeitos da exposição prévia aos crioprotetores, utilizados no presente trabalho, uma vez que não houve diferenças entre os Grupos 2 e 3. Esses resultados indicam que a técnica de vitrificação utilizada com envase dos folículos em capilares de vidro foi satisfatória. SANTOS et al. (2006) encontraram, em ovinos, no grupo controle, expostos aos crioprotetores e criopreservados, $83,1 \%, 62,3 \%$ e $65,7 \%$, respectivamente, de viabilidade folicular, após análise pelo teste do azul 
de tripan. Os resultados encontrados no presente estudo são similares aos de SANTOS et al. (2006); entretanto, esses autores utilizaram método de congelação clássico e estudaram os efeitos de quatro crioprotetores isoladamente (DMSO, etileno-glicol, propanodiol e glicerol), constatando menores índices de viabilidade com o glicerol.

Os crioprotetores apresentam efeitos tóxicos (SCHALKOFF et al., 1989; ViNcent et al., 1990; FUKU et al., 1995), além de causarem choques osmóticos (AGCA et al., 2000). Diferentes alternativas foram propostas para reduzir os efeitos tóxicos dos crioprotetores. A associação de diferentes crioprotetores, passagens de soluções menos concentradas de crioproteores (solução de equilíbrio) para soluções mais concentradas, associação de soluções de crioprotetores menos tóxicas como a sacarose, redução do tempo de exposição à solução crioprotetora (RALL, 1987; ARAV et al., 1993; DHALI et al., 2000; VAJTA et al., 1998), entre outras. Em relação ao tempo de exposição dos folículos pré-antrais isolados, no presente estudo, utilizaram-se 2 minutos (1 minuto na solução 1 e 1 minuto na solução de vitrificação 2 ), tempo que pode ter contribuído para a redução da viabilidade dos grupos de folículos vitrificados. Nesse sentido, a redução do tempo de exposição poderia diminuir os efeitos lesivos encontrados, mostrado pelo teste de viabilidade do azul de tripan. Outro aspecto importante refere-se à concentração dos crioprotetores em folículos pré-antrais. DEMIRCI et al. (2001) estudaram o efeito de várias concentrações $(2 ; 4 ; 6,5 ; 9$; e $10 \mathrm{M})$ de DMSO e Propileno-glicol em ovinos, encontrando altas perdas de viabilidade, em particular com o crioprotetor DMSO, à partir de 6,5 M. Nesse sentido, uma das alternativas para a redução de efeitos tóxicos, encontrada no presente estudo, seria a redução do tempo e também da concentração final da solução de vitrificação.

Em relação ao suporte de envase de ovócitos, micropipetas de vidro têm sido utilizadas para processos de vitrificação; no entanto, com a diferença no presente estudo de serem estiradas por meio de aquecimento, para a redução de seu diâmetro (MEZZALIRA et al., 1999). O vidro apresenta maior condutividade térmica que materiais plásticos utilizados para envase de gametas e embriões. Esse fato proporciona maior velocidade de resfriamento, que é desejável no processo de vitrificação (BUNN et al., 2008). O uso de pipetas de vidro, sem estiramento, como se procedeu no presente estudo, apesar de possivelmente apresentar redução da velocidade de resfriamento, durante o processo de vitrificação, mantém um padrão uniforme dos diâmetros dos capilares nas repetições dos experimentos além de proporcionar maior resistência do capilar no momento do armazenamento no botijão de nitrogênio, evitando perdas por quebras durante a vitrificação/desvitrificação.

No presente estudo, foi utilizado capilar com diâmetro de $1100 \mu \mathrm{m}$ para envase dos folículos por capilaridade. Nesse aspecto, HOCHI et al. (2001) estudaram a vitrificação de ovócitos antrais de bovinos em capilares de vidro com diferentes diâmetros - 2000, 1400, 1000, 630 e $440 \mu \mathrm{m}$-, os quais apresentaram, respectivamente, taxas de resfriamento de 2000, 3000, 5000, 8000 e $12000^{\circ} \mathrm{C} /$ minuto. Os autores não observaram diferenças na porcentagem de estruturas normais após fecundação dos ovócitos, em taxas de resfriamento de $2000(68 \%)$ e $3000^{\circ} \mathrm{C} /$ minuto $(84 \%)$, mas observaram diferença $(\mathrm{P}<0,05)$ destes com ovócitos vitrificados a $12000^{\circ} \mathrm{C} /$ minuto (49\%). É possível que com a alta taxa de resfriamento obtida tenha ocorrido o fenômeno de fratura, que pode ser prevenido reduzindo a taxa de resfriamento, concentrações de crioprotetores e volume da amostra (YAVIN \& ARAV, 2007).

A vitrificação de folículos isolados bovinos é uma alternativa interessante por apresentar baixo custo, praticidade e satisfatórios índices de viabilidade das estruturas foliculares após desvitrificação. Mais pesquisas com essa técnica são fundamentais para o aperfeiçoamento da tecnologia, proporcionando a formação de bancos de gametas femininos.

\section{CONCLUSÃO}

Os resultados obtidos no presente estudo permitem concluir que a redução da viabilidade de folículos pré-antrais, isolados de ovários da espécie bovina, foi devida aos efeitos dos crioprotetores e não pelo processo de vitrificação.

\section{AGRADECIMENTOS}

Agradecemos à FUNDECT pelo suporte financeiro, à UFMS/CNPq/PIBIC pela concessão das bolsas de Iniciação Científica e ao Frigorífico Buriti pelo fornecimento dos ovários.

\section{REFERÊNCIAS}

AGCA, Y.; LIU, J.; RUTLEDGE, J.J.; CRITSER, E.S.; CRITSER, J.K. Effect of osmotic stress on the developmental competence of germinal vesicle and metaphase II stage bovine cumulus oocyte complexes and 
its relevance to cryopreservation. Molecular Reproduction and Development, v.55, p. 212-219, 2000.

AMORIM C.A; RONDINA, D.; LUCCI, C.M.; GIORGETTI, A.; FIGUEIREDO, J.R.; GONÇALVES, P.B. Cryopreservation of sheep primordial follicles. Reproduction in Domestic Animals, v. 42, p.53-57, 2007.

AMORIM, C.A.; RODRIGUES, A.P.R.; RONDINA, D.; FIGUEIREDO, J.R.; GONÇALVES, P.B.D.; GIORGETTI, A. Cryopreservation of ovine primordial follicles using dimethyl sulfoxide. Fertility and Sterility., v.79, p.682-686, 2003.

ARAV, A., RUBINSKY B, FLETCHER G, SEREN E. Cryogenic protection of oocytes with antifreeze proteins. Molecular Reproduction Development, v.36, p.488-93, 1993.

BEGIN, I.; BHATIA, B.; BALDASSARRE, H.; DINNYES A.; KEEFER, C.L. Cryopreservation of goat oocytes and in vivo derived 2- to 4-cell embryos using the cryoloop (CLV) and solid-surface vitrification (SSV) methods. Theriogenology, v.59, p.1839-1850, 2003.

BUNN, S.; CRUZ, F.B.; PEDRAZZI, C.A.F.; BERTOLINI, M.; VIEIRA, A.D.; MEZZALIRA, A. Aumento na sobrevivência após vitrificação de oócitos bovinos imaturos em recipientes com maior condutividade térmica e nitrogênio super-resfriado. Acta Scientiae Veterinariae, v. 36, p. 255-261, 2008.

CELESTINO, J.J.H.; SANTOS, R.R.; LOPES, C.A.P.; MARTINS, F.S.; MATOS, M.H.T.; MELO, M.A.P.; BÁO, S.N.; RODRIGUES, A.P.R.; JOSÉ ROBERTO VIANA SILVA, J.R.V.; FIGUEIREDO, J.R. Preservation of bovine preantral follicle viability and ultra-structure after cooling and freezing of ovarian tissue. Animal Reproduction Science, v.108, p.309-318, 2008.

CHOI, W.J.; YEO, H.J.; LEE, S.A.; LEE, J.H.; PAIK, W.Y. Effect of vitrification method on survivability, follicular growth and ovulation of preantral follicles in mice. Journal Obstetrics Gynaecology Research, v. 33, p. 128-133, 2007.

de la PEÑA, E.C.; TAKAHASHI, Y.; KATAGIRI, S.; ATABAY, E.C.; NAGANO, E. Birth of pups after transfer of mouse embryos derived from vitrified preantral follicles. Reproduction, v.123, p.593-600, 2002.

DEMIRCI, B.; LORNAGE, J.; SALLE, B.; FRAPPART, L.; FRANCK, M.; GUERIN, J.F. Follicular viability and morphology of sheep ovaries after exposure to cryoprotectant and cryopreservation with different freezing protocols. Fertility and Sterility, v. 75, p.124128,2001

DHALI, A.; MANIK, R.S.; DAS, S.K.; SINGLA, S.K.; PALTA, P. Vitrification of buffalo (Bubalus bubalis) oocytes. Theriogenology, v.53, p.1295-1303, 2000.
FIGUEIREDO, J.R.; HULSHOF, S.C.J.; VAN DEN HURK, R.; BEVERS, M.M.; NUSGENS, B.; BECKERS, J.F. Development of a combined new mechanical and enzymatic method for the isolation of intact preantral follicles from fetal, calf and adult bovine ovaries. Theriogenology, v.40, p.789-799, 1993.

FUKU, E.; LIU, J.; DOWNEY, B.R. In vitro viability and ultrastructural changes in bovine oocytes treated with a vitrification solution. Molecular Reproduction and Development, v.40, p.177-185, 1995.

GANDOLFI，F.; PAFFONI，A.; BRAMBILLA， E.P.; BONETTI, S.; BREVINI, T.A.L.; RAGNI, G. Efficiency of equilibrium cooling and vitrification procedures for the cryopreservation of ovarian tissue: comparative analysis between human and animal models. Fertility and Sterility, v. 85, p.1150-1156, 2006.

GUPTA, P.S.P.; NANDI, S.; RAVINDRANATHA, B.M.; SARMA, P.V. In vitro culture of buffalo (bubalus bubalis) preantral follicles. Theriogenology. v.57, p.1839-1854, 2002.

HASEGAWA, A.; HAMADA, Y.; MEHANDJIEV, T.; KOYAMA, K. In vitro growth and maturation as well as fertilization of mouse preantral oocytes from vitrified ovaries. Fertility and Sterility, v.81, p.824-830, 2004.

HOCHI, S.; AKIYAMA, M.; MINAGAWA, G.; KIMURA, K.; HANADA, A. Effects of cooling and warming rates during vitrification on fertilization of in vitro matured bovine oocytes. Cryobiology, v.42, p.6973,2001

ISACHENKO, E.; ISACHENKO, V.; RAMIHI, G.; NAWROTH, F. Cryopreservation of human ovarian tissue by direct plunging into liquid nitrogen. European Journal Obstetrics Gynecology Reproductive Biology, v.108, p.186-193, 2003.

LANDA, V.; TEPLA, O. Cryopreservation of mouse 8cell embryos in microdops. Folia Biologica, v.36, p.153$158,1990$.

LANE, M.; WILLIAM, B.; SCHOOLCRAFT, M.D.; GARDNER, D.K.; Vitrification of mouse and human blastocysts using a novel cryoloop container-less technique. Fertility and Sterility, v.72, p.1073-1078, 1999.

LUCCI, C..M.; KACINSKIS, M.; LOPES, L. H. R.; RUMPF, R.; BÁO, S.N. Effect of different cryoprotectants on the structural preservation of follicles in frozen zebu bovine (Bos indicus) ovarian tissue. Theriogenology, v.61, p.1101-1114, 2004.

LUNA, H.S.; FERRARI, I.; RUMPF, R. Influence of stage of maturation of bovine oocyte at time of vitrification on the incidence of diploid metaphase II at completion of maturation. Animal Reproduction Science, v.68, p.23-28, 2001.

MARTINO, A.; SONGSASEN, N.; LEIBO, S. P. Development intro blastocysts of bovine oocytes 
cryopreserved by ultra-rapid cooling. Biology of Reproduction, v.54, p.1059-1069, 1996.

MATSUMOTO, H.; JYANG, J.Y.; TANAKA, T.; SASADA,H.; SATO,E. Vitrification of large quantities of immature bovine oocytes using nylon mesh. Cryobiology, v.42, n.2, p.139-144, 2001.

MEZZALIRA, A.; VIEIRA, A.D.; CRUZ, F.B.; BARBIERI, D.P.; DAMIANI, J.C. Vitrificação de oócitos bovinos em micropipetas de vidro. Arquivo da Faculdade de Veterinária, v.27, p.262, 1999.

NIEMAN, H. Advances in cryopreservation of bovine oocytes and embryos derived in vitro and in vivo. Arquivo da Faculdade de Veterinária, v.24, p.67-81, 1996.

NUTTINCK, F.; MERMILLOD, P.; MASSIP, A.; DESSY, F. Characterization of in vitro growth of bovine preantral follicles: A preliminary study. Theriogenology, v. 39, p. 811- 812, 1993.

OKTAY, K.; NEWTON, H.; AUBARD, Y.; SALHA, O.; GOSDEN, R.G. Cryopreservation of immature human oocytes and ovarian tissue: an emerging technology. Fertility and Sterility, v.69, p.1-7, 1998.

PARKS, J.E.; RUFFING, N.A. Factors affecting low temperature survival of mammalian oocytes. Theriogenology, v.37, p.59-73, 1992.

RALL, W. F. Factors affecting the survival of mouse embryos cryopreserved by vitritication. Cryobiology, v.24, p.387-402, 1987

SALEHNIA, M. Autograft of vitrified mouse ovaries using ethylene glycol as cryoprotectant. Experimental Animal, v.51, p.509-512, 2002.
SANTOS, R.R; RODRIGUES, A.P.R.; COSTA, S.H.F.; MATOS, M.H.T.; SILVA, J.R.V.; CELESTINO, J.J.H.; MARTINS, F.S.; SARAIVA, M.V.A.; MELO, M.A.P.; FIGUEIREDO, J.R. Teste de toxicidade e criopreservação de folículos pré-antrais ovinos isolados utilizando Glicerol, Etilenoglicol, Dimetilsulfóxido e Propanodiol. Brazilian Journal of Veterinary Research and Animal Science, v.43, p.250-255, 2006.

SCHALKOLFF, E..M.; OSKOWITZ, P.S.; POWERS, R.D. Ultrastructural observations of human and mouse oocytes treated with cryopreservatives. Biology of Reproduction, v.40, p.379-393, 1989.

VAJTA, G.; BOOTH, P. J.; HOLM, P.; GREVE, T.; CALLESEN, H. Successful vitrification of early stage bovine in vitro produced embryos with the open pulled straw (OPS) method. Cryo-Letters, v.18, p.191-195, 1997.

VAJTA, G.; HOLM, M.; BOOTH, P.J.; JACOBSEN, H.; GREVE, T.; CALLESEN, H. Open Pulled Straw (OPS) vitrification: a new way to reduce cryoinjuries of bovine ova and embryos. Molecular Reproduction Development, v.51, p.53-58, 1998.

VINCENT, C.; PICKERING, S.J.; JOHNSON, M. The hardening effect of dimethylsulphoxide on the mouse zona pellucida requires the presence of an oocytes and is associated with a reduction in the number of cortical granules present. Journal of Reproduction and Fertility, v.89, p.253-259, 1990.

YAVIN, S.; ARAV, A. Measurement of essential physical properties of vitrification Solutions. Theriogenology, v. 67 p.81-89, 2007. 